\title{
Interim PET-CT Scan in Advanced Hodgkin's Lymphoma
}

TO THE EDITOR: Johnson et al. (June 23 issue) ${ }^{1}$ report that their trial included 172 patients with advanced-stage Hodgkin's lymphoma who switched from doxorubicin, bleomycin, vinblastine, and dacarbazine (ABVD) to bleomycin, etoposide, doxorubicin, cyclophosphamide, vincristine, procarbazine, and prednisone (BEACOPP) because of positive results on interim positron-emission tomography (PET) with ${ }^{18} \mathrm{~F}$-fluorodeoxyglucose (FDG). Among these patients, the 3-year progression-free survival rate was $67.5 \%$ and the overall survival rate was $87.8 \%$. These patients had more therapy-related adverse events than did patients who had negative results on interim FDG-PET and continued to receive ABVD chemotherapy. Unfortunately, the benefit of early intensification with BEACOPP in patients who had positive results on interim FDG-PET cannot be assessed because, as in other recent nonrandomized studies of chemotherapy intensification, the trial design did not include a randomized comparison between continuation of standard ABVD chemotherapy and intensified BEACOPP chemotherapy and because of futile comparisons with historical cohorts in trials in which the methods and results were not homogeneous. ${ }^{2}$

Remarkably, the German Hodgkin Study Group HD15 trial ${ }^{3}$ showed that 191 patients who continued to have positive findings on FDG-PET after six to eight cycles of BEACOPP had a good 4-year progression-free survival rate of $86.2 \%$ after they received additional treatment with radiation therapy. Comparable results were observed in studies that included patients who had earlystage or advanced-stage Hodgkin's lymphoma and received ABVD. ${ }^{4,5}$ These findings show that radiation therapy can successfully eradicate persistent FDG-avid lesions after first-line therapy and that early intensification of chemotherapy may not be warranted in these patients.

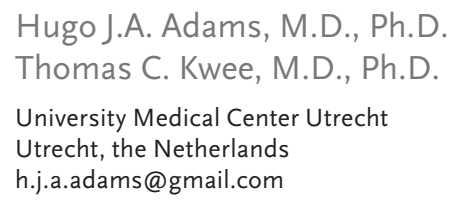

No potential conflict of interest relevant to this letter was reported.

1. Johnson P, Federico M, Kirkwood A, et al. Adapted treatment guided by interim PET-CT scan in advanced Hodgkin's lymphoma. N Engl J Med 2016;374:2419-29.
2. Adams HJ, Nievelstein RA, Kwee TC. Prognostic value of interim FDG-PET in Hodgkin lymphoma: systematic review and meta-analysis. Br J Haematol 2015;170:356-66.

3. Engert A, Haverkamp $\mathrm{H}$, Kobe $\mathrm{C}$, et al. Reduced-intensity chemotherapy and PET-guided radiotherapy in patients with advanced stage Hodgkin's lymphoma (HD15 trial): a randomised, open-label, phase 3 non-inferiority trial. Lancet 2012;379:1791-9. 4. Sher DJ, Mauch PM, Van Den Abbeele A, LaCasce AS, Czerminski J, Ng AK. Prognostic significance of mid- and postABVD PET imaging in Hodgkin's lymphoma: the importance of involved-field radiotherapy. Ann Oncol 2009;20:1848-53.

5. Radford J, Illidge T, Counsell N, et al. Results of a trial of PET-directed therapy for early-stage Hodgkin's lymphoma. N Engl J Med 2015;372:1598-607.

DOI: $10.1056 / N E J M c 1609333$

THE AUTHORS REPLY: Adams and Kwee point out that the patients with positive findings on an interim PET-computed tomographic (PET-CT) scan in our trial were not randomly assigned to continue ABVD. Instead, all these patients switched to BEACOPP regimens. A proposal for this group to undergo randomization was strongly rejected by patient representatives and investigators during the trial design on the grounds that historical series showed unacceptably low rates of progression-free survival ranging from 13 to $28 \%$. By comparison, three separate prospective studies showed that escalation to BEACOPP resulted in progression-free survival rates of 65 to $70 \%{ }^{1,2}$ Further supportive evidence was provided in the Intergroup European Organization for Research and Treatment of Cancer-Lymphoma Study Association-Fondazione Italiana Linfomi H10 (EORTC/FIL H10) trial $^{3}$ in which patients with early-stage disease who continued to have positive findings on an interim PET scan underwent randomization. Although all the patients received consolidation radiotherapy, the rate of progression-free survival at 5 years was higher among patients who received BEACOPP than among patients who continued to receive ABVD ( $91 \%$ vs. $77 \%)$.

Radiotherapy instead of intensified chemotherapy might be appropriate for patients with early-stage disease, but in our trial most patients who had positive PET findings had disease that was too extensive for a limited radiotherapy field. The long-term toxicity of extended-field radiotherapy is well documented ${ }^{4}$ and is a strong reason to use interim PET scanning as a means to tailor therapy to individual patient responses and thereby minimize exposure to radiotherapy. 
Peter Johnson, M.D.

University of Southampton

Southampton, United Kingdom

johnsonp@soton.ac.uk

Judith Trotman, M.D.

Concord Repatriation General Hospital

Sydney, NSW, Australia

Massimo Federico, M.D.

University of Modena and Reggio Emilia

Modena, Italy

Since publication of their article, the authors report no further potential conflict of interest.

1. Gallamini A, Rossi A, Patti C, et al. Interim PET-adapted chemotherapy in advanced Hodgkin lymphoma (HL): results of the second interim analysis of the Italian GITIL/FIL HD0607 trial. Hematol Oncol 2015;Suppl:33s:163. abstract.

2. Press OW, Li H, Schöder H, et al. US intergroup trial of response-adapted therapy for stage III to IV Hodgkin lymphoma using early interim fluorodeoxyglucose-positron emission tomography imaging: Southwest Oncology Group S0816. J Clin Oncol 2016;34:2020-7.

3. Raemaekers J. Early FDG-PET adapted treatment improved the outcome of early FDG-PET positive patients with stages I/II Hodgkin lymphoma (HL): final results of the randomized Intergroup EORTC/LYSA/FIL H10 trial. Presented at the 13th International Conference on Malignant Lymphoma, Lugano, Switzerland, June 17-20, 2015. abstract.

4. Meyer RM, Gospodarowicz MK, Connors JM, et al. ABVD alone versus radiation-based therapy in limited-stage Hodgkin's lymphoma. N Engl J Med 2012;366:399-408.

DOI: 10.1056/NEJMc1609333

\section{Caregivers and Families of Critically III Patients}

TO THE EDITOR: The study by Cameron and colleagues (May 12 issue) $)^{1}$ takes an expanded view of outcomes after critical illness. The authors found that many caregivers have persistent depressive symptoms, which reveal the reverberations of illness beyond the index patient. This article and others ${ }^{2}$ highlight the need to revise the view of the patient from a solitary figure to a person embedded in a social network. A personal network method offers the means to do this. Also called egocentric networks, this approach identifies the various persons around a focal person and elaborates the structure and characteristics of the relationships. ${ }^{3}$ The personal network method explicates how a patient is situated in a complex "social connectome" that is made of strong and weak relationships, kin and non-kin, and persons with varied health habits. It shows the ripples in the network that occur from health shocks such as sudden critical illness, stroke, and myocardial infarction; conversely, network characteristics probably affect outcomes. We look forward to future studies that take a network approach to better understand outcomes and suggest targets for sustainable network recovery.

Amar Dhand, M.D., D.Phil.

Steven K. Feske, M.D.

Brigham and Women's Hospital

Boston, MA

adhand@partners.org

No potential conflict of interest relevant to this letter was reported.

1. Cameron JI, Chu LM, Matte A, et al. One-year outcomes in caregivers of critically ill patients. N Engl J Med 2016;374:1831-41.
2. Adelman RD, Tmanova LL, Delgado D, Dion S, Lachs MS. Caregiver burden: a clinical review. JAMA 2014;311:1052-60.

3. Crossley N, Bellotti E, Edwards G, Everett MG, Koskinen J, Tranmer M. Social network analysis for ego-nets. London: Sage, 2015.

DOI: $10.1056 /$ NEJMc1608225

TO THE EDITOR: The attention to the Perspective article by Wittenberg and Prosser (May 12 issue) ${ }^{1}$ and the study by Cameron et al., which emphasize that a patient's health both affects and is affected by the well-being of family members, is timely. Both articles concern high-income countries with well-developed health systems. We want to stress that challenges for caregivers are even greater in low-income settings. ${ }^{2}$ With the increasing prevalence of chronic noncommunicable diseases, families carry heavy burdens in countries in which health care systems are oriented toward acute care populations. Our research on households in Uganda that include a member with type 2 diabetes has shown the difficulties of providing care. Apart from the high treatment cost and loss of income, household members also experienced stress related to the chronic illness. ${ }^{3}$ Although Wittenberg and Prosser underscore the negative effects on caregivers' health, having a household member with type 2 diabetes may also benefit the cardiometabolic health of other members. ${ }^{3,4} \mathrm{We}$ suggested that diabetes education for patients should be family-based and agree that health is a family affair, not least in low-income countries. 